\title{
QUANTITATIVE HELLY-TYPE THEOREMS
}

\author{
IMRE BÁRÁNY, MEIR KATCHALSKI AND JÁNOS PACH
}

\begin{abstract}
We establish some quantitative versions of Helly's famous theorem on convex sets in Euclidean space. We prove, for instance, that if $C$ is any finite family of convex sets in $\mathbf{R}^{d}$, such that the intersection of any $2 d$ members of $C$ has volume at least 1 , then the intersection of all members belonging to $C$ is of volume $\geq d^{-d^{2}}$. A similar theorem is true for diameter, instead of volume. A quantitative version of Steinitz' Theorem is also proved.
\end{abstract}

1. Introduction. The aim of this paper is to establish some quantitative versions of the following two well-known results on convex sets.

HELLY'S THEOREM (H. T.). If $C$ is a finite family of at least $d+1$ convex sets in $\mathbf{R}^{d}$ and if the intersection of any $d+1$ members of $C$ is nonempty, then $\cap C$ is nonempty.

STEINITZ' THEOREM (S. T.). If $V$ is a set of points in $\mathbf{R}^{d}$ whose convex hull contains a solid ball in $\mathbf{R}^{d}$, then there exists a subset $V^{\prime}$ of $V$ containing not more than $2 d$ points and whose convex hull contains a solid ball with the same center.

Helly's Theorem is not quantitative in the sense that it does not give any information on the size of $\bigcap C$. As a first attempt to get a quantitative version of $H$. T., we suppose that any $d+1$ members of $C$ have a "large" intersection and, then, we try to prove that $\cap C$ is "large". (Here "large" can be meant, for instance, as for large volume or diameter or surface area.) However, no theorem of this type is true. This is shown by the following example. Consider a family $C$ of $2 d$ halfspaces whose intersection is a "small" cube. Then any $2 d-1$ of them have a "large" (unbounded) intersection, while $\cap C$ is "small". Thus, to obtain a quantitative theorem, one has to replace the Helly number $d+1$ by at least $2 d$. In this sense our following theorems are best possible.

"QUANTITATIVE" VOLUME THEOREM (Q.V.T.). There exists a constant $v=$ $v(d)>0$ such that for any finite family $C$ of convex sets in $\mathbf{R}^{d}$; if the intersection of any $2 d$ or fewer members of $C$ has volume at least 1 , then the volume of $\cap C$, is at least $v(d)$.

"QuANTITATIVE" Diameter TheOREM (Q.D.T.). There exists a constant $c=c(d)>0$ such that for any finite family $C$ of convex sets in $\mathbf{R}^{d}$; if the intersection of any $2 d$ or fewer members of $C$ has diameter at least 1 , then the diameter of $\cap C$ is at least $c(d)$.

Received by the editors March 9, 1981 and, in revised form, October 7, 1981.

1980 Mathematics Subject Classification. Primary 52A35.

(c) 1982 American Mathematical Society 0002-9939/81/0000-0101/802.75 
As we shall see later these two theorems are closely related to the following strengthened version of Steinitz' Theorem.

"QUANTITATIVE" STEINITZ TheOREM (Q.S.T.). There exists a constant $r=r(d)>0$ such that for any subset $E$ of $\mathbf{R}^{d}$ whose convex hull contains the $d$-dimensional unit ball $B^{d}$, there exists a subset $F$ of $E$ with $|F| \leq 2 d$ such that the convex hull of $F$ contains a d-dimensional ball of radius $r(d)$ with the same center.

Helly's Theorem appears in [5] (cf. [2]), Steinitz' Theorem appears in [8] (cf. [2] again). A weaker version of our Q.D.T. is implied by a result of B. Grünbaum [3]. (This result states that if the intersection of any $2 d$ members of a finite family $C$ of convex sets in $\mathbf{R}^{d}$ is at least 1-dimensional, then $\bigcap C$ is at least 1-dimensional.) The Q.V.T. appears in [1]. However, an independent proof will be given here, with an explicit constant. In fact, we shall prove Q.V.T., Q.D.T., and Q.S.T. by giving explicit constants $v(d), c(d)$ and $r(d)$.

For related Helly-type results consult $[3,4]$. An excellent survey paper on these matters is [2].

Proofs are given in the next section. The last section contains some remarks.

2. Proofs. The following notation will be used: conv $E$ for the convex hull of $E \subseteq \mathbf{R}^{d}$ and pos $E$ for the smallest convex cone with apex at the origin containing $E, B^{d}$ or $B$ for the unit ball of $\mathbf{R}^{d}$ with center at the origin and $S$ or $S^{d-1}$ for the unit sphere of $\mathbf{R}^{d}, \omega_{d-1}$ for the surface area of $S^{d-1}$ and $c E=\{c x: x \in E\}$ if $c$ is a real number and finally $\|a\|$ denotes the distance from $a \in \mathbf{R}^{d}$ to the origin.

ProOF OF Q.S.T. Assume that $B \subset \operatorname{conv} E$ and consider a regular $d$-dimensional simplex $\Delta$ inscribed in $B$. Since $\Delta \subset \operatorname{conv} E$, each vertex of $\Delta$ is a convex combination of at most $d+1$ elements of $E$, so $\Delta$ is contained in ' $P=\operatorname{conv} E^{\prime}$ where $E^{\prime} \subseteq E$ and $\left|E^{\prime}\right| \leq(d+1)^{2}$. Clearly $\frac{1}{d} B \subset P$ because $\frac{1}{d} B \subset \Delta$. We may suppose without loss of generality that the facets $P_{1}, \ldots, P_{n}$ of $P$ are all simplices (otherwise we can triangulate the nonsimplex facets). It is obvious that

$$
n \leq\left(\begin{array}{c}
(d+1)^{2} \\
d
\end{array}\right)
$$

Since the cones pos $P_{i}(i=1, \ldots, n)$ cover the sphere $S^{d-1}$, for one of them, say $\operatorname{pos} P_{1}$, the surface area of $S \cap \operatorname{pos} P_{1}$ is not less than $\frac{1}{n} \omega_{d-1}$. Let $a \in \operatorname{pos} P_{1}$ be a vector such that the minimal angle between $a$ and the facets of pos $P_{1}$ is maximal. Denote this minimum by $\alpha$. For each facet $L_{i}$ of $\operatorname{pos} P_{1}$ consider the set $D_{i}$ of those vectors whose angle with $L_{i}$ is not larger than $\alpha$ and which lie on the same side of $L_{i}$ as $a(i=1, \ldots, d)$. Clearly $\bigcup_{i=1}^{d} D_{i} \supseteq \operatorname{pos} P_{1}$. Since the surface area of $S \cap D_{i}$ is less than $\omega_{d-2} \operatorname{tg} \alpha$ we get

$$
\begin{aligned}
\frac{1}{n} \omega_{d-1} & \leq \operatorname{Area}\left(S \cap \operatorname{pos} P_{1}\right)<\sum_{i=1}^{d} \operatorname{Area}\left(S \cap D_{i}\right) \\
& <d \omega_{d-2} \operatorname{tg} \alpha .
\end{aligned}
$$

This gives

$$
\alpha>\operatorname{arctg} \frac{\omega_{d-1}}{\mathrm{nd} \omega_{d-2}} .
$$

Now the set $K=\left\{x \in \frac{1}{a} S: \varangle(x, a)=\alpha\right\}$ is contained in $\operatorname{conv}\left(P_{1} \cup\{0\}\right)$ and the vector $(-1 /\|a\|) a$ belongs to the convex hull of 0 and at most $d$ points of $E$. Let $F$ 
be the set of these points and the vertices of $P_{1}$, clearly $|F| \leq 2 d$ and $0 \in \operatorname{conv} F$. The cone with apex $(-1 /\|a\|) a$ and base $K$ is contained in conv $F$ and the origin lies on its axis. It is easily checked that the largest ball contained in this cone (with center at the origin) is $r(d) B$ where

$$
r(d)=\frac{\sin \alpha}{\alpha}\left(1+\frac{1}{d^{2}}+\frac{2}{d} \cos \alpha\right)^{1 / 2}>d^{-2 d}
$$

Since $r(d) B \subset$ conv $F$ this proves the theorem for $r(d)=d^{-2 d}$.

With some additional effort the estimation (2) can be improved to

$$
r(d)>c(2 e d)^{-\lfloor d / 2\rfloor} d^{-2},
$$

where $c$ is a positive constant. This improvement comes mainly from the use of the Upper Bound Theorem [6] instead of the crude estimation (1).

PROOF OF Q.V.T. We suppose that each $C \in C$ is closed. This cannot affect the volumes in question. Every $C \in C$ is the intersection of some closed halfspaces $H_{i}\left(i \in I_{C}\right)$. Let $\mathcal{H}=\left\{H_{i}: i \in I_{C}, C \in C\right\}$. It is clear that the intersection of the interior of any $2 d$ members of $C$ is nonempty, so by Helly's Theorem the interior of $\bigcap C$ is nonempty. We can suppose that $\bigcap C$ is bounded (otherwise it would have infinite volume). Consider the boundary $F$ of the set

$$
G=\left\{x \in R^{d}: \rho(x, \bigcap C) \leq \epsilon\right\}
$$

where $\epsilon>0$ is chosen so small that $\operatorname{vol} G<2 \operatorname{vol}(\cap C)$. Now $F$ is compact and is covered by the open sets $\mathbf{R}^{d} \backslash H(H \in \mathcal{H})$. So, by the Borel covering theorem, there exists a finite subfamily $\sharp^{\prime} \subseteq \mathcal{H}$ such that $F$ is covered by the sets $\mathbf{R}^{d} \backslash H\left(H \in \mathcal{H}^{\prime}\right)$. Now

$$
\bigcap c \subseteq \bigcap H^{\prime} \subset G
$$

so in order to prove the theorem it suffices to prove the following lemma.

LEMMA. Suppose that for a finite family $\sharp$ of halfspaces of $\mathbf{R}^{d}$ the intersection $P=\bigcap \mathcal{H}$ is a polytope with unit volume. Then there exists a subfamily $\mathcal{H}^{\prime} \subseteq \mathcal{H}$ with $\left|H^{\prime}\right| \leq 2 d$ such that vol $\cap \mathcal{H}^{\prime} \leq 1 / v(d)$.

Proof. Consider the ellipsoid $E$ of largest volume contained in $P$ (with center 0, say). Then as it is well known (see e.g. [9]) $P \subset d E$. Using a volume-preserving linear transformation we can suppose that $E$ is a ball. Then

$$
\operatorname{vol}(d E)=d^{d} \operatorname{vol} E \leq d^{d} \operatorname{vol} P=d^{d},
$$

so that $P \subset \rho B$ where

$$
\rho^{d} \operatorname{vol} B=d^{d} .
$$

Using polarity we get $(\rho B)^{*}=\frac{1}{\rho} B \subset P^{*}$. Then, on applying Q.S.T., there exists an at most $2 d$-membered subset, $Q$, of the vertices of $P^{*}$ such that

$$
P^{*} \supseteq \operatorname{conv} Q \supset \frac{r(d)}{\rho} B \text {. }
$$

Using polarity again

$$
\left(\frac{r(d)}{\rho} B\right)^{*}=\frac{\rho}{r(d)} B \supset(\operatorname{conv} Q)^{*} \supseteq P^{* *}=P
$$


and clearly, $(\operatorname{conv} Q)^{*}$ is the intersection of $|Q| \leq 2 d$ members of $\not H$. Denoting the set of these halfspaces by $\sharp^{\prime}$ we get

$$
\operatorname{vol}\left(\bigcap \varkappa^{\prime}\right)=\operatorname{vol}(\operatorname{conv} Q)^{*} \leq\left(\frac{\rho}{r(d)}\right)^{d} \operatorname{vol} B \leq d^{2 d^{2}} \text {. }
$$

This gives that the theorem (and the lemma) are true with $v(d)=d^{-2 d^{2}}$. (Again, this can be improved by (3).)

PROOF OF Q.D.T. FIRST PROOF. It is again easily seen that the theorem follows from the special case when $C$ consists of a finite number of closed halfspaces and $\cap C$ is a convex polytope with diameter 1 . But this special case is the dual of Q.S.T. The result is that $c(d) \geq \frac{1}{2} r(d)$ where $r(d)$ comes from (2) or (3). This, again, can be improved to $c(d) \geq[\sqrt{(d+1) / 2 d}] r(d)$ using the following consequence of Helly's Theorem (see [2, Theorem 2.6]). If $X \subset \mathbf{R}^{d}$ and diam $X=1$, then $X$ can bé covered by a ball of radius $(d / 2(d+1))^{1 / 2}$.

SECOND PROOF. (This proof does not use Q.S.T. and gives a smaller constant $c(d)$ than the first proof. As a matter of fact, we want to have as large $c(d)$ as possible.) We shall show that if $\operatorname{diam} \cap C \leq 1$, then diam $\cap C^{\prime} \leq r_{0}^{-1}(d)$ for some (at most) $2 d$-membered subfamily $C^{\prime}$ of $C$. By H.T., $\cap C \neq \emptyset$, suppose it contains the origin. Then $\bigcap C \subset B$ and $B \subset \Delta$ where $\Delta$ is a regular simplex with side (or, what is the same, diameter) $\sqrt{2 d(d+1)}$. Consider the $d+1$ halfspaces $H_{0}, H_{1}, \ldots, H_{d}$ whose intersection is $\Delta$. For each $H_{i}$ there exist (at most) $d$ members of $C$ whose intersection lies in $H_{i}$ (by H.T., again). Thus there exists an at most $d(d+1)$ membered subfamily $C^{\prime \prime}$ of $C$ such that $\bigcap C^{\prime \prime} \subset \Delta$, whence diam $C^{\prime \prime} \leq \sqrt{2 d(d+1)}$.

Now we reduce the number of elements of $C^{\prime \prime}$ to $2 d$. This is done through repeated applications of the following lemma.

LEMMA. Let $k \geq 2 d$ and $C_{1}, \ldots, C_{k+1}$ be given convex sets in $\mathbf{R}^{d}$ such that $0 \in \bigcap_{1}^{k+1} C_{i}$ and each $D^{j}=\bigcap_{i=1 ; i \neq j}^{k+1} C_{i}$ contains a point $a_{j}$ with $\left\|a_{j}\right\|=1(j=$ $1, \ldots, k+1)$. Then $\bigcap_{1}^{k+1} C_{i}$ contains a point a with $\|a\| \geq h$ where $h=h(d, k)>0$ depends only on $d$ and $k$.

PROOF OF THE LEMMA. The distance of a spherical cap $P$ is the distance of its convex hull from the origin. Clearly there exists an $h=h(d, k)$ so that for a spherical cap $P$ with distance $h$

$$
\frac{\text { Area } P}{\omega_{d-1}}>\frac{d}{k+1}
$$

where we used the assumption $k \geq 2 d$. For each $a_{j}$ consider the spherical cap $P\left(a_{j}\right)$ with center $a_{j}$ and distance $h$. By our choice of $h$ there exists a point $b \in S^{d-1}$ common to at least $d+1$ such caps, otherwise each point of $S^{d-1}$ is covered by at most $d$ caps and

$$
d \geq \frac{\sum_{j} \operatorname{Area} P\left(a_{j}\right)}{\omega_{d-1}}>\frac{(k+1) d}{k+1}=d .
$$

This, in turn, implies that the spherical cap $P(b)$ with distance $h$ and center $b$ contains at least $d+1$ points from the set $\left\{a_{1}, \ldots, a_{k+1}\right\}$, suppose these points are $a_{1}, \ldots, a_{d+1}$. The line segment $\left[0, a_{j}\right]$ meets the bounding hyperplane $H$ of $P(b)$ in the point $b_{j}(j=1, \ldots, d+1)$, obviously $b_{j} \in D^{j}$. By Radon's theorem (see [7 
or 2]), applied in the hyperplane $H$ to the points $b_{1}, \ldots, b_{d+1}$, there exists a point $a \in H$ common to $D^{1}, D^{2}, \ldots, D^{d+1}$. Consequently $a \in \bigcap_{i=1}^{k+1} C_{i}$. On the other hand, $a \in H$ implies $\|a\| \geq h$.

To get an estimation for $r_{0}(d)$ in the case $d \geq 3$ observe that

$$
h(d, k)=\frac{k+1-2 d}{k+1}
$$

satisfies the requirements of the Lemma. So we have

$$
\begin{aligned}
r_{0}(d) & \geq \frac{1}{\sqrt{2 d(d+1)}} \prod_{k=2 d}^{d(d+1)-1} \frac{k+1-2 d}{k+1} \\
& =(1+o(d)) \sqrt{2 \pi} d^{-2 d-1 / 2} 2^{2 d} e^{-2 d}
\end{aligned}
$$

This is less than the $r(d)$ from Q.S.T.

3. Remarks. 1. The Q.V.T. and Q.D.T. remain true if instead of the finiteness of $C$ we suppose that the members $C$ are all closed and one of them is compact.

2. Let $v^{*}(d)\left(c^{*}(d)\right.$ and $\left.r^{*}(d)\right)$ denote the supremum of those $v(d)(c(d)$ and $r(d))$ for which Q.V.T. (Q.D.T. and Q.S.T.) holds. What we have shown is that

$$
v^{*}(d) \geq d^{2 d^{2}}, \quad c^{*}(d) \geq r^{*}(d) \sqrt{(d+1) / 2 d}
$$

and

$$
r^{*}(d) \geq d^{-2 d}
$$

(or a somewhat better estimation from (3)). We have not been able to determine the order of magnitude of these functions. We think that $v^{*}(d) \approx d^{-c_{1} d}$ and $r^{*}(d) \approx$ $c_{2} d^{-1 / 2}$ where $c_{1}$ and $c_{2}$ are positive constants.

3. Let $\operatorname{vol}_{n} C$, the $n$-dimensional volume of $C$ be the supremum of the area of the intersection of $C$ with a $n$-dimensional flat. (Here $C$ is a convex set in $\mathbf{R}^{d}$.) It follows from (4), that there exist constants $K(d, n)>0(n=1,2, \ldots, d)$ such that if a finite family $C$ of convex sets has the property that the intersection of any $2 d$ or fewer members of $C$ is of $n$-dimensional volume at least 1 , then

$$
\operatorname{vol}_{n}(\bigcap c) \geq K(d, n) \text {. }
$$

This result is somewhat stronger than Q.V.T. and Q.D.T.

\section{REFERENCES}

1. I. Bárány, M. Katchalski and J. Pach, Helly's theorem, Amer. Math. Monthly (submitted).

2. L. Danzer, B. Grünbaum and V. Klee, Helly's Theorem and its relatives, Proc. Sympos. Pure Math., vol. 7, Amer. Math. Soc., Providence, R. I., 1963, pp. 101-180.

3. B. Grünbaum, The dimensions of intersections of convex sets, Pacific J. Math. 12 (1962), 197202.

4. H. Hadwiger, H. Debrunner and V. Klee, Combinational geometry in the plane, Holt, Rinehart \& Winston, New York, 1964.

5. E. Helly, Über Mengen konvexer Körper mit gemeinschaftlichen Punkten, Jber. Deutsch. Math.Verein. 32 (1923), 175-176.

6. P. McMullen and G. C. Shephard, Convex polytopes and the upper bound conjecture, Cambridge Univ. Press, New York, 1971.

7. J. Radon, Mengen konvexer Körper, die einen gemeinsamen Punkt enthalten, Math. Ann. 83 (1921), 113-115. 
8. E. Steinitz, Bedingt konvergente Reihen und konvexe Systemes. I-II-III, J. Reine Angew. Math. 143 (1913), 128-175, 144 (1914), 1-40, 146 (1916), 1-52.

9. F. John, Extremum problems with inequalities as subsidiary conditions, Courant Anniversary Volume, Interscience, New York, 1948, pp. 187-204.

Mathematical Institute, Hungarian ACADEMY OF SCienCes, 1053 Budapest, Hungary (Current address of Imre Bárány and János Pach)

DEPARTMENT OF MATHEMATICs, UNIVERsity of ALBERTA, EDMONTON, Alberta, CaNADA T6G 2G1

Current address: (Meir Katchalski): Department of Mathematics, Technion, Haifa, Israel 